\title{
„Secundum vocem concordare, sensu tamen discrepare.“ Der Streit um die Deutung des Aristoteles an der Universität Ingolstadt im späten 15. und frühen 16. Jahrhundert
}

Wie bekannt, nahm im 15. Jahrhundert der Streit um die Deutung des Aristoteles die Form einer Debatte zwischen Nominalisten und Realisten an, die sich unmittelbar im Unterricht an den Universitäten niederschlug und in der modernen Forschung als ,Wegestreit' bezeichnet wird. ${ }^{1}$ So eindeutig der Begriff des Wegestreits die Tatsache der Auseinandersetzung zum Ausdruck bringt, so kompliziert sind die Motive, die zu diesem Streit geführt haben. Es lohnt sich, diese Motive aufzudecken, denn sie geben Auskunft nicht nur über die mannigfaltige Art und Weise, in der die Texte des Aristoteles im späten Mittelalter gelesen wurden, sondern auch über die verschiedenen methodischen Ausgangspunkte, die dabei eine Rolle spielten.

Wichtige Dokumente zur Geschichte des Wegestreits wurden von Franz Kardinal Ehrle im Anhang zu seiner Studie über Petrus de Candia ediert. ${ }^{2}$ Die meisten dieser Texte haben in der Forschung bereits vielfach Beachtung gefunden, wie etwa das Antwortschreiben der Kölner Universität an die Kurfürsten aus dem Jahre 1425 oder die Verteidigungsschrift der Pariser Nominalisten von 1474. ${ }^{3}$ Eine weitere Sammlung von Fragmenten und Texten aus diesem Anhang wurde bis jetzt jedoch kaum untersucht. Es handelt sich hierbei um Schriftstücke aus der Handschrift München, Universitätsbibliothek, $2^{\circ}$ Cod. ms. 482, die Bezug nehmen auf die Diskussionen zwischen Nominalisten und Realisten an der Universität Ingolstadt, in deren Mittelpunkt die Frage nach dem korrekten Umgang mit den Texten des Aristoteles stand. ${ }^{4}$ Auf diese Schriftstücke möchte ich im Folgenden mein Hauptaugenmerk richten. Die Handschrift selbst ist inzwischen verloren gegangen. ${ }^{5}$ Die von Ehrle edierten

$1 \mathrm{Vgl}$. Zénon Kaluza, Les querelles doctrinales à Paris. Nominalistes et réalistes aux confins du XIV et du $X V^{e}$ siècles (Quodlibet 2), Bergamo 1988, und Götz-Rüdiger Tewes, Die Bursen der Kölner ArtistenFakultät bis zur Mitte des 16. Jahrhunderts (Studien zur Geschichte der Universität zu Köln 13), Köln 1993. 2 Vgl. Franz Kardinal Ehrle, Der Sentenzenkommentar des Peters von Candia, des Pisaner Papstes Alexanders V. Ein Beitrag zur Scheidung der Schulen in der Scholastik des 14. Jahrhunderts und zur Geschichte des Wegestreits, Münster 1925, S. 281-342.

3 Vgl. Erich Meuthen, Die alte Universität (Kölner Universitätsgeschichte 1), Köln 1988, S. 174-175, und Zénon Kaluza, „La crise des années 1471-1482. L'interdiction du Nominalisme par Louis XI“, in: Maarten J. F. M. Hoenen / J. H. Josef Schneider / Georg Wieland (Hgg.), Philosophy and Learning. Universities in the Middle Ages (Education and Society in the Middle Ages and Renaissance 6), Leiden 1995, S. 293-327.

4 Ehrle, Der Sentenzenkommentar des Peters von Candia, op. cit., S. 326-342.

5 Sie gilt als Kriegsverlust; vgl. Die lateinischen mittelalterlichen Handschriften der Universitätsbibliothek München. Die Handschriften aus der Filioreihe (Die Handschriften der Universitätsbibliothek München 3/2), beschr. von Natalia Daniel / Gerhard Schott / Peter Zahn, Wiesbaden 1979, S. 60. Eine partielle Beschreibung geben Carl Prantl, Geschichte der Ludwig-Maximilians-Universität in Ingolstadt, Landshut, 
Partien vermitteln jedoch ein repräsentatives Bild der Vorgänge in Ingolstadt, das auch in anderen Quellen gezeichnet wird: Die Nominalisten waren dort in der Mehrzahl und dominierten die Artesfakultät, während die Realisten versuchten, diese Vorherrschaft zu durchbrechen und deshalb die Art und Weise anprangerten, in der die Nominalisten den Text des Aristoteles auslegten. Die Realisten betrachteten die Vorgehensweise der Nominalisten als grundsätzlich verschieden von der ihrigen und stellten sie als eine Gefahr für den Glauben dar. ${ }^{6}$ Ihrer Meinung nach gab es nicht nur Unterschiede zwischen beiden Lehren, sondern in vielen Punkten auch Widersprüche, so dass an ein Zusammengehen beider Wege nicht zu denken war. ${ }^{7}$

Bemerkenswert ist, dass die Nominalisten ihrerseits den Unterschied zu den Realisten als inhaltlich unbedeutend herunterspielten. Sie betrachteten ihre Auslegung des Aristoteles als die gängige Praxis. Was sie darunter verstanden, geht aus den von Ehrle edierten Textstïcken und aus den Werken Ingolstädter Nominalisten wie Johannes Parreut und Johannes Eck klar hervor. Es handelte sich um eine Deutung, die, wenn erforderlich, neue Begriffe oder Unterscheidungen einführte, die als solche im Text des Aristoteles zwar nicht enthalten waren, sich damit jedoch in Einklang bringen ließen. Die Nominalisten gingen also selbstständig mit dem Text des Aristoteles um und sahen darin die eigentliche Aufgabe des Auslegers, wie sie im Unterricht an der Universität erfüllt werden sollte, auch von den Realisten.

Genau diese Praxis wurde von den Realisten jedoch in Frage gestellt. In einer ausführlichen Liste zählten sie Punkte auf, in denen sie sich von den Nominalisten unterschieden. ${ }^{8}$ Dabei wurde immer wieder hervorgehoben, dass die Deutung der Nominalisten mit dem Text des Aristoteles im Widerspruch oder gegen dessen Intention sei. So hätten die Nominalisten eine elfte Kategorie und zusätzliche Bestimmungen des Begriffs ,Qualität' eingeführt, die Aristoteles nie erwähnt habe. ${ }^{9}$ Aus einer weiteren von Ehrle edierten Liste geht hervor,

München, 2 Bde., München 1872, Bd. I, S. 126, Anm. 85, und Ehrle, Der Sentenzenkommentar des Peters von Candia, op. cit., S. 327-330.

6 Die Debatten in Ingolstadt werden erörtert in Prantl, Geschichte der Ludwig-Maximilians-Universität, op. cit., Bd. I, München 1872, S. 52-54, S. 77-84 und S. 126-129; Ehrle, Der Sentenzenkommentar des Peters von Candia, op. cit., S. 187-193; Astrik L. Gabriel: „Via antiqua and via moderna and the Migration of Paris Students and Masters to the German Universities in the Fifteenth Century", in: Albert Zimmermann (Hg.), Antiqui und Moderni. Traditionsbewußtsein und Fortschrittsbewußtsein im späten Mittelalter (Miscellanea Mediaevalia 9), Berlin 1974, S. 439-483, bes. S. 476-478, und Arno Seifert, Die Universität Ingolstadt im 15. und 16. Jahrhundert. Texte und Regesten (Ludovico Maximilianea. Quellen 1), Berlin 1973, S. 45-48 und S. 67-70.

7 Als Beleg führten die Realisten an, dass die Nominalisten an mehreren Universitäten nicht zugelassen würden, was nicht der Fall wäre, wenn es nur ein und dieselbe Lehre gäbe (,si esset una et eadem doctrina in artibus“); vgl. Ehrle, Der Sentenzenkommentar des Peters von Candia, op. cit., S. 327 (Anhang).

8 Ibid., S. 334-338 (Anhang): „Secuntur positiones et dicta rationalis et naturalis philosophie, in quibus dicta modernorum plerumque discrepant et contradicunt doctrine Realium [...].“

9 Ibid., S. 335-336 (Anhang): „Tertio principaliter discrepant a doctrina Aristotelis et Realium doctorum circa librum predicamentorum Aristotelis. Et primo, ut dictum est, ponentes undecim predicamenta [...] contra intentionem Aristotelis et omnium Peripateticorum. [...] item circa species qualitatis, ubi Nominales plures species nominant, quas Aristoteles non posuit.“ 
warum dies für die Realisten so problematisch war. ${ }^{10}$ Sie zeigte auf, dass die nominalistische Deutung dazu führte, dass man die Lehre des Glaubens nicht mehr mit Hilfe von Aristoteles verteidigen konnte. Hielt man zum Beispiel mit den Nominalisten daran fest, dass sich die Relation von der Substanz nicht unterscheidet, dann sei man nicht im Stande zu erklären, wie die göttlichen Personen, die der Substanz nach eins sind, sich durch ihre gegenseitigen Beziehungen (relationes) voneinander unterscheiden, wie es die Kirche lehre. ${ }^{11}$

Offensichtlich hatten die Realisten an einem Aristoteles Interesse, dessen Lehre mit dem Glauben zu vereinbaren war. Nun ist der Ausgangspunkt dieser Haltung nicht ausreichend erklärt, wenn man annimmt, es ginge hier lediglich um die Harmonie zwischen Glauben und Vernunft. Es spielte noch eine andere historische Gegebenheit eine Rolle. In den Augen vieler Realisten bedeutete das Studium der aristotelischen Werke, wie es in den im Jahre 1366 im Auftrag von Urban VI. erneuerten Statuten der Pariser Universität vorgesehen war, die kirchliche Approbation des Aristoteles. ${ }^{12}$ Dies wurde auch ausdrücklich hervorgehoben: „ecclesia catholica doctrinam Aristotelis [...] probavit." ${ }^{\text {"13 }}$ Im Umkehrschluss argumentierten einige Realisten, dass es sich überall dort, wo die Auslegung der aristotelischen Texte im Widerspruch zum Glauben stand, nicht um den authentischen Aristoteles handeln könne. Der Glaube gab so den Maßstab für die richtige Deutung des Philosophen ab. ${ }^{14}$

Diese Strategie wurde auch von den Realisten in Ingolstadt aufgegriffen. Sie versuchten damit, die Richtigkeit der Lehre ihrer Gewährsmänner wie Thomas von Aquin, Albertus Magnus und Duns Scotus unter Beweis zu stellen. Diese hätten sich immer an Aristoteles gehalten und dessen Schriften bei der Erklärung des Glaubens benutzt. Deshalb sei ihre

10 Ibid., S. 338-342 (Anhang). Diese Liste ist überschrieben, ibid., S. 338 (Anhang): „Infra notantur positiones et dicta [...], que videntur contrariare dictis sanctorum patrum et discrepare ab his, que tenet ecclesia katholica."

11 Ibid., S. 338 (Anhang): „Tenet ecclesia quod relationibus persone divine distinguuntur, cui contrariari videntur, qui relationem a sua substantia non distinguunt nec aliis fundamentis, que sunt qualitates et quantitates.“

12 Dazu Luca Bianchi, Censure et liberté intellectuelle à l'Université de Paris (XIII ${ }^{e}-X I V^{e}$ siècles), Paris 1999, S. 159-162. Vgl. Chartularium Universitatis Parisiensis, hg. von Henricus Denifle / Aemilio Châtelain, Bd. III, Paris 1894, S. 143-148 (n. 1319), bes. S. 145.

13 Vgl. Zénon Kaluza, „Les débuts de l'Albertisme tardif (Paris et Cologne)“, in: Maarten J. F. M. Hoenen I Alain de Libera (Hgg.), Albertus Magnus und der Albertismus. Deutsche philosophische Kultur des Mittelalters (Studien und Texte zur Geistesgeschichte des Mittelalters 48), Leiden 1995, S. 207-295, bes. S. 233, Anm. 14, und Bianchi, Censure et liberté intellectuelle, op. cit., S. 310, Anm. 129.

14 So behauptete Lambert von 's Heerenbergh in seiner Quaestio de salvatione Aristotelis, die um 1489 bei Heinrich Quentell in Köln gedruckt wurde (Freiburg UB, Ink $4^{\circ} \mathrm{D} 1586$ ), fol. $\mathrm{Bi}^{\mathrm{rb}}$, die völlige Übereinstimmung zwischen Aristoteles und dem Glauben: „[...] Aristoteles in nullo doctrinae aut legi Christi contrariatur, immo est ei per omnia conformis.“ Dazu Hans Gerhard Senger, „Was geht Lambert von Heerenberg die Seligkeit des Aristoteles an?", in: Albert Zimmermann (Hg.), Studien zur mittelalterlichen Geistesgeschichte und ihren Quellen (Miscellanea Mediaevalia 15), Berlin 1982, S. 293-311, bes. S. 300-301. Der gängige Vorbehalt, dass Aristoteles in einigen Punkten, wie der Ewigkeit der Welt, der Lehre der Kirche widersprach, ist hier verschwunden; vgl. etwa Lambert von 's Heerenbergh, ibid., fol. Bii ${ }^{\text {va }}$, Et sic patet quod concorditer loquutus est [sc. Aristoteles, $\mathrm{MH}$ ] cum sanctis doctoribus, qui concedunt mundum per naturalem motum non incepisse, sed per creationem a Deo.“ Thomas habe in diesem Punkt, so fügt Lambert hinzu, die Lehre des Aristoteles voll und ganz vertreten: ibid., fol. Bii ${ }^{\mathrm{va}}:$ „[...] in hoc per omnia concordat sanctus Tho$\operatorname{mas}[\ldots]$.. 
Lehre immer als „sana, solida, salubris, secura“ betrachtet worden. ${ }^{15}$ Die Realisten folgten dieser Lehre und somit sei auch ihre Lehre als mit Aristoteles konform aufzufassen. ${ }^{16}$

Die Nominalisten reagierten darauf mit dem Hinweis, dass sich bei diesen Lehrern auch falsche Deutungen des Aristoteles finden ließen, was die Realisten zu einer zweiten Strategie zwang, die der ersten entgegengesetzt war, sie aber ergänzen sollte. Dabei spielte der Ruf der Gewährsmänner eine ausschlaggebende Rolle. Nach den Realisten komme Thomas von Aquin, Albertus Magnus und Duns Scotus eine solche Autorität in der Kirche zu, dass deren Auffassung auch in Fällen, wo sie eine bestimmte Lehre nicht weiter begründeten, dennoch zu gelten habe, da sie unendlich viele sichere Thesen gelehrt hätten. ${ }^{17}$ Ganz anders sehe es bei den Gewährsmännern der Nominalisten aus, die keinen solchen Ruhm hätten und von denen man deshalb eher annehmen könne, sie hätten sich in ihrer Auslegung des Aristoteles geirrt. ${ }^{18}$

Die Realisten fühlten sich also in zweierlei Hinsicht in ihrem interpretatorischen Umgang mit Aristoteles bestärkt. Sie hielten sich erstens an dessen Text, der kirchlich approbiert war, ohne ihn zu ändern, und stützten sich zweitens auf Autoritäten, von denen fest stehe, dass sie von der Lehre der Kirche nicht abgewichen seien. Thomas von Aquin, Albertus Magnus und Duns Scotus seien somit die besten Ausleger des Aristoteles, denn sie hätten bereits im Voraus genau das getan, was durch die spätere kirchliche Approbation des

15 Ehrle, Der Sentenzenkommentar des Peters von Candia, op. cit., S. 334 (Anhang): „[...] doctrina Alberti Magni, S. Thome de Aquino, Egidii de Roma, Alexandri de Ales, Scoti, Bonaventure, Richardi, Petri de Tarantasio, Petri de Pallude aliorumque Realium doctorum maxime conformis habetur dictis Aristotelis, ubi potest salva fide, ideoque semper sana, solida, salubris, secura reperta est.“ Wie aus dem Zusatz „salva fide“ hervorgeht, hielt man in Ingolstadt zumindest dem Wortlaut nach am alten Vorbehalt gegenüber Aristoteles fest. Inhaltlich jedoch ist davon keine Spur mehr zu finden.

16 Ibid., S. 329 (Anhang): „Si beatus Thomas, Albertus Magnus, Egidius de Roma, Alexander de Hallis, Scotus, Bonaventura et alii Realium doctores errant, erramus, imo totus Predicatorum et Minorum ordo, eorum imitatores." Der Verweis auf die Ordenstraditionen, der die Richtigkeit der Lehre noch weiter bestätigen soll, findet sich auch im Antwortschreiben der Kölner Universität an die Kurfürsten aus dem Jahre 1425; siehe ibid., S. 285 (Anhang): „Denique hec ipsa inhibitio [sc. vom Realismus, MH] non parvum honoris prejudicium inferret preclaris illis Ordinibus, de quorum fuerunt professione sepedicti Doctores [sc. Thomas von Aquin, Albertus Magnus und Duns Scotus, MH].“ Unsere Handschrift enthielt auch Dokumente, die nicht aus Ingolstadt stammten, wie das Edikt Ludwigs XI. gegen die Pariser Nominalisten aus dem Jahre 1474, das in zwei Abschriften vertreten war. Das Antwortschreiben der Kölner Universität war jedoch nicht enthalten, trotz der Übereinstimmung in der Argumentation, wie aus dem Inhaltsverzeichnis bei Ehrle, ibid., S. 327-330, hervorgeht.

17 Ibid., S. 329 (Anhang): „Sed non est credibile predictos gloriosos doctores, quorum doctrina semper sana et secura comperta est, errare, quorum etiam tanta in ecclesia auctoritas est, ut si dictorum suorum nullam afferent rationem, cum tamen pene infinitas solidissimas afferant, ipsi nos sua auctoritate frangerent.“

18 Ibid., S. 329 (Anhang): „Presumendum potius est Marsilius, Wuridanum, Hockam, Dorp, Brinkl, Biligam ceterosque, et nomine et auctoritate inglorios, errasse et non modo ipsos sed et eorum sequaces, quos Nominales seu Terministas vocant." Auch hier ist der Bezug zum Schreiben der Kölner Universität aus dem Jahre 1425 deutlich. Der Ruhm von Marsilius und Buridan sei in keiner Weise mit dem von Thomas und Scotus zu vergleichen, wie unter Berufung auf das Gewissen hervorgehoben wird; ibid., S. 285 (Anhang): „Eya, quomodo dictat alicuius recta conscientia, quod ubi [...] Buridanus, Marcilius et alii simplices Magistri Artium sunt accepti, ibi tam insignes sacre Theologie Professores proscribantur?" Dieses Argument trifft jedoch insofern sachlich nicht zu, als Marsilius auch Professor der Theologie war. Womöglich handelt es sich hier um ein altes Argument, das bereits zirkulierte, bevor Marsilius 1394 den Doktortitel in Heidelberg erwarb. 
Aristoteles zum Ausdruck gebracht wurde, nämlich aufzudecken, dass dessen Lehre mit dem Glauben nicht im Widerspruch stehe.

Die Nominalisten replizierten auf diese Taktik der Realisten, indem sie deren Autoritätsglauben einer scharfen Kritik unterzogen. Dieser wurde sogar als deren Hauptfehler herausgestellt, aus dem alle ihre Fehldeutungen des Aristoteles hervorgingen. Mit mehreren Verweisen auf das Decretum Gratiani hoben die Nominalisten hervor, dass die Reputation einer Person nichts über die Wahrheit ihrer Lehre aussage und nicht bewirken dürfe, dass man ihr eher als einer anderen Glauben schenke. ${ }^{19}$

Diesem Autoritätsglauben stellten die Nominalisten den Gebrauch der Vernunft entgegen, mit dem sie sich von den Realisten zu unterscheiden suchten. Sie wollten nichts ohne Vernunftgründe annehmen und sahen darin auch ein besonderes Merkmal ihrer Schule. ${ }^{20}$ Als Beispiel dafür, wie sinnlos der Autoritätsglauben bei der Suche nach Wahrheit sei und in welche Widersprüche man beim Festhalten an Autoritäten geriet, verwiesen sie auf die Universalienfrage. Es gebe unter den Realisten keine Einstimmigkeit über die Natur der Universalien. Sie seien vielmehr so zerstritten, dass jeder von ihnen eine andere Interpretation vertrete. Zudem hätten sie sich in der Deutung des Thomas von Aquin von einem neuen Kollegen korrigieren lassen, der ihnen gezeigt habe, dass sie Thomas' Schriften nicht verstanden hätten. Wie könne man sich unter solchen Umständen auf die Autorität des Thomas von Aquin berufen? Es seien also die Reales, nicht die Nominales, die vom Weg der Wahrheit abgewichen sind. ${ }^{21}$

19 Die Haltung der Nominalisten gegenüber den Realisten war belehrend und überheblich. Wie aus dem Verweis auf die Heiligkeit (sanctitas) hervorgeht, stand vor allem die Verehrung des Thomas von Aquin im Mittelpunkt der Kritik; siehe Ehrle, ibid., S. 332 (Anhang): „Quoniam vero erranti veritatis viam ostendere opus creditur pietatis, facere non potuimus, quin principium et caput erroris ipsis Antiquis aperiremus, quod auctorum vetustati sanctitatique vite nimis tribuunt; $a b$ eo omnis eorum profiscitur error, quod ne facerent eos satis admonere deberet, quod sanctitas persone non facit, ut citius credatur ei [...]." Es folgt darauf ein Hinweis auf eine Stelle aus dem Decretum Gratiani, aus dem hervorgeht, dass die Heiligkeit nicht genügt, sondern weitere Gründe für die Bedeutung eines Autors als Autorität vorliegen sollten; vgl. Decretum Magistri Gratiani 1, 9, 5 (Corpus Iuris Canonici 1), hg. von Aemilius Richterus / Aemilius Friedberg, Leipzig 1879, S. 17: „Alios autem [sc. scriptores, MH] ita lego, ut quantalibet sanctitate doctrinaque polleant, non ideo uerum putem, quia ita ipsi senserunt, sed quia mihi per alios auctores, uel canonicas uel probabiles rationes, quod a uero non abhorreat, persuadere potuerunt." Weitere Hinweise auf das Decretum schließen sich an.

20 Ehrle, Der Sentenzenkommentar des Peters von Candia, op. cit., S. 332 (Anhang): „Haec omnia ostendunt, Antiquos vehementer errare, Modernos vero, qui sine ratione nichil asserunt, pure veritatis viam et sequi et tenere."

21 Vgl. ibid., S. 332 (Anhang). Der neue Kollege wird in der Transkription von Ehrle als ,frater Petrus Sirarcz" angegeben. Ehrle kann über diese Person nichts Weiteres finden und vermutet, dass es sich hier um einen Franziskaner handelt, da, wie er meint, ,die Predigerbrüder in Ingolstadt nicht ansässig waren“. Tatsächlich aber studierten Dominikaner an der Universität Ingolstadt, wie etwa die beiden Brüder Georg und Peter Schwartz. Letzterer ist der Autor des berühmten Clypeus Thomistarum, einer Verteidigung der Lehre des Thomas. Offensichtlich ist dieser Petrus Schwartz der gemeinte neue Kollegen und Ehrle hat den Namen einfach falsch transkribiert. Über die Namensform ,Schwartz' siehe meine Studie Speculum philosophiae medii aevi. Die Handschriftensammlung des Dominikaners Georg Schwartz († nach 1484) (Bochumer Studien zur Philosophie 22), Amsterdam 1994, S. 18-20. Zu den Dominikanern in Ingolstadt siehe ibid., S. 1619 und S. 25-26. Informationen zu Petrus Schwartz gibt B. Konrad Vollmann, „Nigri (Schwarz), Petrus“, in: Die deutsche Literatur des Mittelalters. Verfasserlexikon, Bd. VI, Berlin ${ }^{2} 1987$, S. 1008-1013. Petrus 
In den Exercitata veteris artis des Ingolstädter Nominalisten Johannes Parreut wurden weitere Argumenten dafür angeführt, dass die Lehre der Realisten unmöglich stimmen könne. ${ }^{22}$ Parreut versuchte, die Realisten mit ihren eigenen Argumenten zu treffen, indem er unterstrich, dass die berühmtesten Theologen gänzlich ohne die Annahme von realen Universalien ausgekommen seien, auch dort, wo es um die Auslegung des Glaubens ging. ${ }^{23}$ Die berühmtesten Theologen waren für ihn jedoch nicht Thomas von Aquin und Duns Scotus, sondern Johannes Gerson, Peter von Ailly und Wilhelm von Ockham, deren Ruhm zur Zeit über die ganze Welt verbreitet sei, wie Parreut mit einem Seitenhieb gegen die Realisten hervorhob, die auf die Verbreitung der Lehre von Thomas und Scotus gesetzt hatten. ${ }^{24} \mathrm{Er}$ begründete seine Wahl damit, dass die zeitgenössischen Gelehrten den älteren vorzuziehen seien, vor allem wo es um Fragen der Logik geht, denn der zeitgenössische Geist sei schärfer als der alte. ${ }^{25}$ Der Verweis auf Autoritäten sei also erlaubt, wenn er der Vernunft nicht entgegenstehe. Aus dieser Perspektive entschuldigte er auch Thomas von Aquin. Man könne Thomas seinen Irrtum in der Universalienfrage nicht ankreiden, denn zu seiner Zeit war diese Frage noch nicht so ausführlich diskutiert worden, dass er sich darüber ein richtiges Urteil hätte bilden können.

Entscheidender noch war der Hinweis Parreuts auf die Verurteilung der realistischen Universalienlehre auf dem Konzil von Konstanz (1414-1418). ${ }^{26}$ Wie der anonyme Autor der

Schwartz wurde 1473 in Ingolstadt immatrikuliert; vgl. Die Matrikel der Ludwig-Maximilians-Universität Ingolstadt - Landshut - München, hg. von Götz Freiherr von Pölnitz, München 1937, S. 34: „Frater Petrus Swartz de Cadana ordinis predicatorum sacre theologie baccalarius formatus." Daraus ergibt sich eine Datierung des oben genannten Vorfalls auf ca. 1473.

22 Johannes Parreut, Exercitata veteris artis, Ingolstadt 1492 (München, Bayerische Staatsbibliothek, Inc. $931 \mathrm{~m}$ ), fol. aii ${ }^{\mathrm{v}}$-aviii ${ }^{\mathrm{v}}$. Johannes Parreut (Bairreit, Bairrewt) ließ sich 1474 in Ingolstadt immatrikulieren; vgl. Die Matrikel der Ludwig-Maximilians-Universität, op. cit., S. 54. Er war Doktor der Medizin und Leibarzt der Herzoge Ludwig des Reichen und Georg des Reichen und starb 1495 an der Pest. Zu ihm und seinen Exercitata siehe Carl Prantl, Geschichte der Logik im Abendlande, Bd. IV, Leipzig 1870, photomechanischer Nachdruck Graz 1955, S. 239-240, und Prantl, Geschichte der Ludwig-Maximilians-Universität, op. cit., Bd. I, S. 76, und Bd. II, S. 483-484. Weitere Literatur ist verzeichnet in Die Matrikel der LudwigMaximilians-Universität Ingolstadt - Landshut - München, Teil 1, Bd. IV/1, hg. von Götz Freiherr von Pölnitz / Laetitia Boehm, München 1981, S. 60, s.v. ,Parreut‘.

23 Johannes Parreut, Exercitata, op. cit., fol. aiiii": „[...] famosissimi theologi etiam omnia melius sanabant, etiam ea quae fidem tangunt, sine illis universalibus, quam alii qui ea posuerunt [...].“

24 Johannes Parreut, ibid., fol. aiii": „Pollicear me denique secuturum in illa materia illos moderniores doctores, videlicet cancellarium Johannem Gerson, doctorem christianissimum, et Petrum Cameracensem et strennuum pugilem veritatis Guilhelmum Occan, quorum fama et laus quasi nunc per totum orbem est diffusa."

25 Ibid., fol. aiii": „Et non sine ratione illos imitor, quoniam de quanto doctores fuerunt moderniores de tanto solidius scripsisse, praecipue in artibus, et ingeniis subtilioribus floruisse eos arbitror.“"

$26 \mathrm{Ibid}$., fol. $\left[\mathrm{av}^{\mathrm{T}-\mathrm{v}}\right]$ : „Damnata est novissime inter errores Hus et Heronimi per celeberrimum Concilium Constantiense positio ista de universalium realium et aeternorum positione." Parreut zitiert hier wörtlich aus einem Brief des Johannes Gerson, Gerson à un Frère Mineur (Lyon, 7 décembre 1426) (Euvres Complètes, Bd. II), hg. von Mgr. Palémon Glorieux, Paris 1960, S. 276-280 (n. 58), bes. S. 278. Auch die Realisten verwiesen auf Gerson, und zwar am Anfang der oben in Anm. 8 genannten Liste, in der sie die Unterschiede zwischen Realisten und Modernen auf den Punkt brachten. Sie zitierten aus der Schrift De examinatione doctrinarum, in der Gerson ausführt, dass jede Lehre (doctrina) zuerst danach zu beurteilen sei, ob sie mit dem Glauben übereinstimme; vgl. Ehrle, Der Sentenzenkommentar des Peters von Candia, op. cit., S. 334 (Anhang) und Johannes Gerson, De examinatione doctrinarum (Euvres Complètes, Bd. IX), hg. von Mgr. 
Ingolstädter Replik an die Realisten, argumentierte Parreut mit einer juristischen Quelle. Er habe die Akten des Konzils eingesehen und festgestellt, dass Hieronymus von Prag den Satz „homo, leo et asinus non sunt naturae communes“ zurücknehmen musste. ${ }^{27}$ Damit habe die Kirche sich in der Universalienfrage für den Nominalismus und gegen den Realismus entschieden. Denn der Nominalismus lehre, dass der Mensch im Allgemeinen alle Menschen sind (,homo in communi sunt omnes homines“), so Parreut. ${ }^{28}$ Darüber hinaus zeigte er mit Verweisen auf Stellen aus der Metaphysik, der Zweiten Analytik und der Kategorienschrift, dass diese Deutung der Universalien vollkommen in Übereinstimmung mit der Lehre des Aristoteles sei..$^{29}$ Der nominalistische Aristoteles stand also nicht im Widerspruch zum Glauben, wie Parreut seinem Leser zu verstehen gab.

Aus dieser Entgegnung Parreuts geht hervor, dass es den Nominalisten nicht um eine Kritik an Aristoteles oder der kirchlichen Autorität ging. ${ }^{30}$ In dieser Hinsicht unterschieden sich die Nominalisten nicht von den Realisten und eben deshalb konnten die Nominalisten in Ingolstadt verneinen, dass es einen wirklichen Unterschied zu den Realisten gab. In der Praxis sah die Lektüre des Aristoteles jedoch ganz anders aus, wo bei dessen Auslegung erhebliche Differenzen hervortraten, wie das Beispiel der Universalienlehre gezeigt hat. Dieser doppeldeutige Umstand führte die Realisten dazu zu behaupten, dass die Nominalisten zwar dem Wortlaut nach mit den Realisten übereinstimmten, dem Sinn nach jedoch eine ganz andere Lehre vertraten. ${ }^{31}$ Die Frage, die sich hierbei stellt, ist, was genau mit diesem unterschiedlichen Sinn gemeint war. Um dies herauszufinden, möchte ich ein Beispiel aus der Liste von Differenzpunkten, die von den Realisten erstellt wurde, im weiteren Verlauf meines Beitrags aufgreifen, und zwar die Frage, ob man nach Aristoteles neben den zehn bekannten noch eine elfte Kategorie annehmen kann. Nach den Realisten nahmen die Nominalisten eine solche Kategorie an, die sie als ,praedicamentum signi“ bezeichneten, jedoch habe Aristoteles nie davon gesprochen; auch sei dies gegen die Absicht des Aristoteles und aller Peripatetici. ${ }^{32}$ Zuerst ist es also wichtig festzustellen, was genau die Texte des Aristoteles zur Anzahl der Kategorien behaupten. Danach möchte ich auf das Verständnis dieser Anzahl im Laufe des 13. und 14. Jahrhunderts eingehen, damit deutlich wird, in welchem Zusammenhang der Begriff des „praedicamentum signi“ erstmals auftaucht, um schließlich zu den Debatten in Ingolstadt zurückzukehren.

Palémon Glorieux, Paris 1973, S. 456-475 (n. 456), S. 465. Die Autorität des Gerson wurde also von beiden Seiten zur Stärkung der je eigenen Position eingesetzt.

27 Johannes Parreut, Exercitata, op. cit., fol. [av ] : „Et ego eadem acta et actitata eiusdem Concilii vidi, ubi Heronimus revocat quod homo, leo et asinus non sunt naturae communes.“

$28 \mathrm{Ibid}$., fol. [av']: „Dico quod universale reale in essendo est idem realiter cum omnibus suis individuis et singularibus simul sumptis. Et sunt a parte rei species rerum, ut homo in communi sunt omnes homines, scilicet Petrus et Paulus et sic de singulis.“

29 Vgl. ibid., fol. [av'].

30 Ausdrücklich betont Parreut, die nominalistische Universalienlehre sei in Übereinstimmung mit der ,intentio Peripateticorum“ (fol. aiiii") und der ,auctoritas Ecclesiae“ (fol. av ${ }^{\mathrm{v}}$ ).

31 Ehrle, Der Sentenzenkommentar des Peters von Candia, op. cit., S. 338 (Anhang): „Et si quandoque secundum vocem concordant cum doctrina Realium, sensu tamen discrepant.“

32 Ibid., S. 335 (Anhang): „[...] ponunt undecimum predicamentum, quod appellant predicamentum signi, cuius Aristoteles nunquam meminit." Siehe auch das Zitat oben Anm. 9. 
Aristoteles hatte an vielen Stellen seines Werkes die Kategorien erwähnt, aber er tat dies nicht immer auf dieselbe Art und Weise. ${ }^{33}$ Vor allem zur Anzahl der Kategorien waren seine Ausführungen sehr unterschiedlich. Wo er sich ausdrücklich darüber aussprach, behauptete er, dass es endlich viele Kategorien gebe, so in den Analytica posteriora, oder dass es deren zehn gebe, so in der Topica. ${ }^{34}$ In der Kategorienschrift selbst zählte er zehn Kategorien auf, sagte aber nichts Weiteres über deren Anzahl. ${ }^{35}$ Dies führte in den Kommentaren zu seinen Werken zwangsläufig zur Frage, ob es in der Tat eine definitive Anzahl von eben zehn Kategorien gebe oder ob deren Anzahl geringer oder sogar größer sei. ${ }^{36}$

Weiter wurde diskutiert, was von Aristoteles durch die Kategorien klassifiziert wurde, wobei am meisten umstritten war, ob es sich um Begriffe oder um Sachen aus der extramentalen Wirklichkeit handelte, oder um beides. Im Hintergrund stand hier die Trennung zwischen wirklichem und gedanklichem Seienden, die Aristoteles in seiner Metaphysik angesprochen hatte. ${ }^{37}$ Auch spielte der Umstand eine Rolle, dass im lateinischen Text der Kategorienschrift von ,eorum quae dicuntur" gesprochen wurde, was darauf hinzudeuten schien, dass es sich um Begriffe oder Wörter handelte, wohingegen in den Übersetzungen der $\mathrm{Me}$ taphysik von "secundum se vero esse“ die Rede war, das jeweils verschiedentlich durch die verschiedenen Kategorien bezeichnet wurde, was nahe legte, dass es sich auch um Verschiedenes in der extramentalen Wirklichkeit handle. ${ }^{38}$

33 Vgl. die hilfreiche Auflistung der Stellen in Aristoteles, Kategorien (Werke in deutscher Übersetzung 1/1), übers. u. erl. von Klaus Oehler, Berlin 1984, S. 289-292. Zur Kategorienlehre des Aristoteles siehe Lambert M. de Rijk, Aristotle. Semantics and Ontology, Vol. I: General Introduction. The Works on Logic (Philosophia Antiqua 91/1), Leiden 2002, S. 358-471, und neuerdings mit Verweisen auf weitere Literatur Ludger Janses, Art. „katêgoria / Kategorie“ in: Otfried Höffe (Hg.), Aristoteles-Lexikon, Stuttgart 2005, S. 299-304.

34 Aristoteles, Analytica posteriora II, 22, 83b15-17, und Topica I, 9, 103b22-23.

35 Aristoteles, Categoriae 4, 1b25-27.

36 Vgl. etwa Johannes Buridanus, Quaestiones in Praedicamenta 3 (Veröffentlichungen der Kommission für die Herausgabe ungedruckter Texte aus der mittelalterlichen Geisteswelt 11), hg. von Johannes Schneider, München 1983, S. 14-29: „Utrum sint decem et non plura praedicamenta.“ Und Johannes Versor, Quaestiones super totam veterem artem Aristotelis, Köln 1494, unveränderter Nachdruck Frankfurt am Main 1967, Praedicamenta, fol. $29^{\mathrm{va}}-30^{\text {rb }}$ : „Quaeritur utrum tantum sunt decem praedicamenta.“

37 Aristoteles, Metaphysica V 7, 1017a22-35. Vgl. Thomas von Aquin, De ente et essentia 1 (Opera omnia, Bd. XLIII), Rom 1976, S. 369: „Sciendum est igitur quod, sicut in V Methaphisice Philosophus dicit, ens per se dupliciter dicitur: uno modo quod diuiditur per decem genera, alio modo quod significat propositionum ueritatem." Siehe auch Les Auctoritates Aristotelis. Un Florilège Médiévale (Philosophes Médiévaux 17), hg. von Jacqueline Hamesse, Louvain/Paris 1974, Metaphysica (155), S. 128: „Ens per se aliud est in anima, aliud extra animam [...].“

$38 \mathrm{Vgl}$. Aristoteles Latinus, Categoriae 4, 1b26, hg. von Laurentius Minio-Paluello, Brügge 1961, S. 6 (translatio Boethii), S. 86 (translatio Guillelmi); Aristoteles Latinus, Metaphysica V, 7, 1017a22-23, translatio anonyma sive ,media', hg. von Gudrun Vuillemin-Diem, Leiden 1976, S. 94, und Aristoteles Latinus, Metaphysica V, 7, 1017a22-23, recensio et translatio Guillelmi de Moerbeka, hg. von Gudrun VuilleminDiem, Leiden 1995, S. 103. Die Deutung, dass es sich um Begriffe handle, konnte sich auf den Kommentar des Boethius stützen; vgl. Manlius Severinus Boethius, In Categorias Aristotelis libri quatuor 1 (Patrologia Latina 64), hg. von Jacques-Paul Migne, Paris 1847, Sp. 180C: „Adeo non de rebus, sed de vocibus tractaturus est, ut diceret ,dicuntur'. Res enim proprie non dicuntur, sed voces [...].“ 
In der Regel wurden diese Fragen in den Kommentaren zur Ars vetus erörtert, und zwar in dem Teil, der der Kategorienschrift gewidmet war. ${ }^{39}$ Dabei sollte festgehalten werden, dass es sich hier um die erste Stelle handelte, an der gemäß der im Unterricht befolgten Sequenz der aristotelischen Schriften der Begriff ,Kategorie“ verwendet wurde. ${ }^{40} \mathrm{Zudem}_{\text {gilt }}$ zu bedenken, dass im Lehrplan vieler Universitäten die Lektüre von Petrus Hispanus als Einleitung zum aristotelischen Organon vorgesehen war. ${ }^{41}$ Petrus Hispanus behandelte die Kategorien in seinem Tractatus im dritten Teil, der eine Bearbeitung der Kategorienschrift in der Übersetzung von Boethius darstellte. Er hatte die Stelle, an der Aristoteles die zehn Kategorien nannte und von ,eorum quae dicuntur" sprach, wörtlich übernommen. ${ }^{42}$ Aus der Perspektive des Studienverlaufs war also bereits zu Beginn den Studenten vermittelt worden, dass es sich um eine Menge von zehn Kategorien handelte und dass die von den Kategorien klassifizierten Gegenstände als Begriffe oder Wörter zu interpretieren waren. In den Texten, die später im Unterricht behandelt wurden, sah die Sache anders aus. In der Physik und der Metaphysik wurden nirgends zehn Kategorien, sondern immer weniger aufgezählt; auch drückte sich Aristoteles dort vielfach so aus, als ob es sich um die Einteilung von extramentalen Gegenständen handle. ${ }^{43}$

Wie stark die Idee der Zehnzahl war - auch dort, wo Aristoteles weniger Kategorien aufzählte - geht aus dem Metaphysikkommentar des Thomas hervor. An einer Stelle im fünften Buch, wo Aristoteles nur acht Kategorien aufgezählt hatte, sprach Thomas in seinem Kommentar von zehn. Es handelte sich hier um die Stelle, wo Aristoteles hervorhob, dass auf verschiedene Weisen vom Seienden gesprochen werden könne. Thomas fasste den Text des Aristoteles folgendermaßen zusammen: „primo distinguit ens, quod est extra animam, per decem praedicamenta. ${ }^{\text {(44 }}$

39 Andere Stellen, die zur Diskussion der Problematik führten, werden genannt bei Giorgio Pini, „Scotus on Deducing Aristotle's Categories“, in: Joël Biard / Irène Rosier-Catach (Hgg.), La Tradition Médiévale des Catégories (XII $-X V^{e}$ Siècles) (Philosophes Médiévaux 45), Louvain-la-Neuve 2003, S. 23-35, bes. S. 24. Dem Aufsatz von Giorgio Pini verdanke ich wichtige Einsichten in die Problematik sowie mehrere Quellenangaben.

40 Das erste aristotelische Werk, über das an den Artesfakultäten gelesen wurde, war die Kategorienschrift; vgl. etwa Hugo Ott / John M. Fletcher, The Mediaeval Statutes of the Faculty of Arts of the University of Freiburg im Breisgau, Notre Dame 1964, S. 40 (de libris audiendis) und S. 55 (quo tempore anni quivis librorum veniat legendus). Die Statuten der Artesfakultät zu Ingolstadt erwähnen ebenfalls die Ars vetus als erste Gruppe aristotelischer Schriften; vgl. Johannes Mederer, Annales Ingolstadiensis Academiae, Bd. IV, Ingolstadt 1782, S. 69-94, bes. S. 93. Die in der Kategorienschrift behandelten Kategorien wurden im Unterricht nicht als ,categoriae", sondern als ,praedicamenta ' bezeichnet. Es wurden jedoch auch andere Begriffe wie ,genera', ,principia“ oder ,generalissima‘ verwendet; vgl. die Zitate in den Anmerkungen 37 (Thomas), 47 (Albertus Magnus) und 56 (Johannes Duns Scotus) .

$41 \mathrm{Ott} /$ Fletcher, The Mediaeval Statutes, op. cit., S. 40 und S. 55.

42 Petrus Hispanus, Tractatus called afterwards Summule Logicales III, 5 (Philosophical Texts and Studies 22), hg. von Lambert M. de Rijk, Assen 1972, S. 29: „Eorum que secundum nullam complexionem dicuntur singulum aut significat substantiam aut quantitatem aut qualitatem aut ad aliquid aut ubi aut quando aut situm esse aut habere aut facere aut pati.“

43 Siehe die Tabelle in Aristoteles, Kategorien, op. cit., S. 289-292.

44 Thomas von Aquin, In duodecim libros Metaphysicorum Aristotelis expositio V, 9, hg. von M.-R. Cathala / Fr. Raymundus M. Spiazzi, Turin 1964, S. 238 (n. 889). Etwas zuvor sprach Thomas, Bezug nehmend auf die gleiche Stelle, ebenfalls von zehn Kategorien; ibid., S. 237 (n. 885): „Quod ex hoc patet, quia ipse 
Wie aus diesem Zitat ersichtlich wird, hielt Thomas nicht nur an der Zehnzahl fest, sondern ging auch davon aus, dass die Kategorien das extramentale Sein klassifizierten. Diese Einteilung geschah ihm zufolge auf der Grundlage der verschiedenen Arten der Prädikation. Auf diese Weise brachte er die logische und die metaphysische Bedeutung der Kategorien miteinander in Verbindung. Er tat dies, weil nach ihm Seiendes nicht durch Seiendes näher bestimmt werden könne, denn als Seiendes füge dieses keine weitere Bestimmung zum Seienden hinzu. Die Bestimmung könne deshalb nur begrifflich mit Hilfe der Prädikation geschehen. Diese Prädikation sei jedoch nicht willkürlich, denn sie bringe die verschiedenen Arten des Seienden zum Ausdruck. ${ }^{45}$

Ich habe diese Stelle von Thomas nicht nur erwähnt, um deutlich zu machen, wie selbstverständlich für ihn die Zehnzahl war und wie er versuchte, die logische und die metaphysische Bedeutung der Kategorien zusammenzubringen, sondern auch, weil es sich bei ihm um einen Autor handelt, auf den die Realisten des 15. Jahrhunderts zurückgriffen, wenn sie behaupteten, dass es nach Aristoteles nur zehn Kategorien gab und nicht mehr. ${ }^{46}$

Diese Selbstverständlichkeit wurde von einem anderen Gewährsmann der Realisten, von Albertus Magnus, in seinem Kommentar zu den Kategorien mit einem Beweis bekräftigt. Nach Albertus hatten viele vor ihm versucht, Argumente zu finden, um zu beweisen, dass es in der Tat nur zehn Kategorien gebe, oder wie er sich auch ausdrückte: zehn ,prima principia rerum". Er wollte dazu auch selbst einen Versuch machen, indem er aus den zu diesen „prima principia“ gehörigen Arten der Prädikation deren Zehnzahl ableitete. Dazu teilte er die möglichen Arten der Prädikation erschöpfend auf und stellte dann fest, dass es in der Tat genau zehn seien. ${ }^{47}$

Der Beweis von Albertus wurde von Johannes Versor in seinem Kommentar zur Ars vetus ausführlich zitiert. Er sprach in diesem Zusammenhang von der „sufficientia venerabilis domini Alberti“", durch die bewiesen werde (probatur), dass es in der Tat nur zehn Kategorien gebe. ${ }^{48}$ Damit galt für ihn die Anzahl, die in der Kategorienschrift aufgeführt wurde, als

postmodum, ens secundum se dividit in decem praedicamenta [...].“ Vgl. Aristoteles, Metaphysica V, 7, 1017a25-27.

45 Thomas von Aquin, ibid. Vgl. John F. Wippel, „Thomas Aquinas's Derivation of the Aristotelian Categories (Predicaments)“, in: Journal of the History of Philosophy 25 (1987), S. 13-34, und Pini, „Scotus on Deducing Aristotle's Categories“, op. cit., S. 25-28.

46 So etwa die Thomisten in Köln; vgl. Copulata in veterem artem secundum viam doctoris Thomae de Aquino, Praedicamenta, Köln 1494 (Freiburg UB, Ink 4 D 1421 c), fol. 47va: „Quaeritur utrum tantum decem sint praedicamenta. Dicendum quod sic. [...] patet per sufficientiam quam tangit sanctus Thomas [...]. Est etiam alia sufficientia quam ponit doctor sanctus quinto Metaphysicae [...].“

47 Albertus Magnus, Liber de Praedicamentis I, 7 (Opera omnia, Bd. I), hg. von Augustus Borgnet, Paris 1890, S. 163-164: ,[...] multi laboraverunt ut rationem ponant quare numerus principiorum omnium rerum stat in decem, nos, quantum possumus, studebimus ex propriis horum generum modis praedicandi ostendere hujus numeri rationem. " Bereits in der Antike wurde ein derartiger Versuch von Simplicius in dessen Kommentar zur Kategorienschrift unternommen. Simplicius verweist auch auf andere Versuche. Eine Übersetzung dieses Textes lag im Jahre 1266 aus der Hand des Wilhelm von Moerbeke vor; vgl. Simplicius, Commentaire sur les Catégories d'Aristote. Traduction de Guillaume de Moerbeke (Corpus Latinum Commentariorum in Aristotelem Graecorum V/1), hg. von Adriaan Pattin / W. Stuyven, Louvain 1971, S. 90-92, bes. S. 91: „Talis utique quaedam erit et a nobis consolatio pertractata desiderantibus accipere decem genera per divisionem indefectibilem [...].“ Zur Datierung siehe ibid., S. xi.

48 Johannes Versor, Quaestiones super totam veterem artem, Praedicamenta, op. cit., fol. $29^{\mathrm{vb}}-30^{\mathrm{ra}}$. 
begründet. So konnte er dann auch denjenigen, die für eine geringere oder größere Anzahl argumentiert hatten, entgegenhalten, dass dies im Widerspruch zum Text des Aristoteles stand: ,in oppositum est Philosophus in textu. ${ }^{49}$ Aus den Schriftstücken in der Handschrift München, Universitätsbibliothek, $2^{\circ}$ Cod. ms. 482, geht hervor, dass die Werke von Thomas von Aquin, Albertus Magnus und Johannes Versor im Unterricht in Ingolstadt studiert wurden. ${ }^{50}$ Es ist also anzunehmen, dass die dortigen Realisten sich auf derartige Überlegungen stützten, wenn sie behaupteten, dass nach Aristoteles die Anzahl der Kategorien auf zehn beschränkt sei. ${ }^{51}$

Wie gesagt, vertraten die Nominalisten in Ingolstadt die Ansicht, dass die Zehnerliste durch eine elfte Kategorie zu ergänzen sei. Aristoteles hatte jedoch an keiner Stelle in seinen Schriften von einer elften Kategorie gesprochen. Deshalb waren die Ingolstädter Realisten sicher, dass die Nominalisten eine falsche Interpretation gaben, und griffen sie so heftig an. Es gab jedoch bereits im 14. Jahrhundert Überlegungen, ob die Anzahl nicht eine andere als zehn sein könne. So hob Ockham hervor, dass die Zehnzahl der Kategorien schwierig zu beweisen sei, obwohl alle wichtigen Autoren daran festhielten. ${ }^{52}$ Franciscus de Mayronis wies kurze Zeit später in seinen Passus super Universalia et Praedicamenta Aristotelis et Perihermenias darauf hin, dass die Zehnzahl nicht selbstverständlich und auf keinen Fall als „per se notum“ zu betrachten sei, wie einige behaupteten. Dass man sie beizubehalten habe, wie gemeinhin gefordert wurde, sei lediglich eine Sache des Autoritätsglaubens, der getragen werde von der Tatsache, dass die Mehrheit diese Anzahl annehme: ,quia omnes hodie supponunt decem esse praedicamenta, ipsa non sunt neganda, et tunc habetur sola fides pro

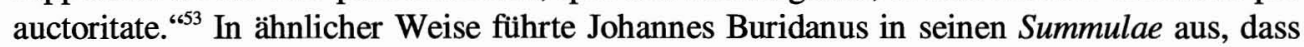

49 Ibid., fol. $29^{\mathrm{va}}$.

50 Die Realisten wiesen darauf hin, dass die Nominalisten hervorgehoben hätten, auch sie würden die Werke der genannten Autoren mit den Studenten lesen; vgl. Ehrle, Der Sentenzenkommentar des Peters von Candia, op. cit., S. 327.

51 Eine Handschrift mit dem Kommentar des Johannes Versor zur Ars vetus wird genannt im Bibliothekskatalog der Artesfakultät von 1492; siehe Mittelalterliche Bibliothekskataloge Deutschlands und der Schweiz, Bd. III/1, bearb. von Paul Ruf, München 1932, S. 233-256, bes. S. 250. Es handelt sich dabei um die Handschrift München, Universitätsbibliothek $2^{\circ}$ Cod. ms. 557, die auch den Kommentar zur Logica nova enthält; vgl. Die lateinischen mittelalterlichen Handschriften der Universitätsbibliothek München, op. cit., S. 79-78.

52 Wilhelm von Ockham, Expositio in Librum Praedicamentorum Aristotelis 7, 2 (Opera Philosophica, Bd. II), hg. von Gedeon Gál, St. Bonaventure, NY, 1978, S. 161: „[...] difficile est probare quod sint tantum decem praedicamenta." Und id., Summa logicae I, 41 (Opera Philosophica, Bd. I), hg. von Philotheus Boehner / Gedeon Gál / Stephanus Brown, St. Bonaventure, NY, 1974, S. 114: „Ponuntur autem ab omnibus auctoribus decem praedicamenta [...].“

53 Franciscus de Mayronis, Passus super Universalia et Praedicamenta Aristotelis et Perihermenias (Logicalia), Toulouse 1490, fol. [Dviii ${ }^{\mathrm{va}-\mathrm{vb}}$ ]. Zu diesem Traktat, den Franciscus am Ende seines Lebens verfasst hat, also um 1328, ohne ihn gänzlich fertig zu stellen (der Kommentar zu De interpretatione ist unvollendet geblieben), siehe P. Bartholomäus Roth, Franz von Mayronis O.F.M. Sein Leben, seine Werke, seine Lehre vom Formalunterschied in Gott (Franziskanische Forschungen 3), Werl in Westfalen 1936, S. 173-179. Das Werk ist in mehreren Handschriften und Frühdrucken überliefert und trägt dort verschiedene Titel, unter anderem ,Super Praedicabila et Praedicamenta und ,Logicalia (so im hier zitierten Druck Toulouse 1490). Wie er am Anfang seines Werkes hervorhebt, versucht Franciscus zu zeigen, dass es zwischen der Wahrheit der Philosophie und der Autorität der göttlichen Weisheit keinen Widerspruch gibt; siehe ibid., fol. Aiir ${ }^{a}$ : ,[...] ad ostendendam quod veritas philosophiae non contradicat auctoritati divinae sapientiae decrevi passus totius philosophiae discurrere [...]. “ So auch im oben genannten Fall der Kategorien, mit dem er aufdecken 
Aristoteles die Zehnzahl nicht begründet habe.$^{54}$ Buridanus selbst hielt an der Zehnzahl fest, die er als ,modi praedicandi non reducibiles“ betrachtete, denn diese sei von Aristoteles so bestimmt worden, wenn auch nicht bewiesen. Aber, so fügte er in seinem Kommentar zur Kategorienschrift hinzu, ,non invenimus, quod ipse dixit, quod non sint plura“. Für Buridanus schloss die faktische Zahl von zehn also nicht aus, dass es im Sinne des Aristoteles noch weitere Kategorien geben könnte, zum Beispiel, wenn zusätzliche „modi praedicandi“ gefunden würden, die sich nicht auf die bekannten zehn zurückführen ließen. ${ }^{55}$

Buridanus hatte keine weiteren „modi praedicandi“ genannt. Es gab jedoch schon länger Überlegungen, die in Richtung einer neuen Kategorie wiesen. In dieser Hinsicht ist der Kommentar zur Ars vetus des Johannes Duns Scotus eine wichtige Quelle. Dort wurde als eines der Argumente angeführt, dass die Logik von Begriffen handle, die Begriffe bezeichnen, und dass diese Begriffe als einer besonderen Kategorie zugehörig aufgefasst werden sollten, neben den Begriffen, die auf die Wirklichkeit verweisen, wie es in der Metaphysik geschehe. Für den Logiker würden somit elf Kategorien bestehen, für den Metaphysiker dagegen nur zehn. ${ }^{56}$ Scotus selbst behielt die Zehnzahl bei. ${ }^{57}$ Aber in der Zeit nach Scotus wurde die Möglichkeit einer elften Kategorie, wie sie in dem von Scotus angeführten Argument erwogen wurde, aufgegriffen, und zwar von Marsilius von Inghen.

Marsilius unterschied in seinem Kommentar zur Ars vetus ebenfalls zwischen Begriffen und Wörtern, die für Dinge in der Wirklichkeit stehen, und Begriffen und Wörtern, die auf Begriffe und Wörter verweisen, wie etwa der Begriff ,genus` für die Begriffe ,Mensch ‘ und ,Lebewesen“ steht. Letztere wurden im Einklang mit der Tradition als Begriffe der zweiten

möchte, dass die Autorität in der Philosophie ähnlich wie im Glauben eine Rolle spielt, denn wenn sich kluge Leute Aristoteles anschließen, auch in den Fällen, wo es keine Beweise gibt, dann sollte man sich doch noch viel mehr Christus anschließen, dem der Vater alles offenbart hat; siehe ibid., fol. [Dviii $\left.{ }^{\mathrm{vb}}\right]:$, [...] si intellectui Aristotelis gratis se subiugaverunt tot sollertes, ut consentirent in his, quae nulla ratione probata sunt [...], multo magis est subiiciendus intellectus iugo Christi, cui Pater omnia revelavit.“ Anders als Buridanus, Johannes Parreut und Johannes Eck fragte Franciscus sich jedoch nicht, ob es mehr, sondern ob es weniger Kategorien als zehn geben könnte. Wie Roth, Franz von Mayronis, op. cit., S. 179, festgestellt hat, wurde die Schrift im 14. und 15. Jahrhundert oft zitiert. Die Mehrzahl der erhaltenen Handschriften stammt aus dem 15. Jahrhundert.

54 Johannes Buridanus, Summulae in Praedicamenta 3.1.8 (Artistarium 10-3), hg. von Egbert P. Bos, Nijmegen 1994, S. 18: „Et sciendum est quod numquam Aristoteles posuit rationem ad ostendendum quod non essent alia praedicamenta praeter ista decem."

55 Johannes Buridanus, Quaestiones in Praedicamenta 3, op. cit., S. 19. Vgl. auch id., Summulae in Praedicamenta, op. cit., S. 122 (Appendix I).

56 Johannes Duns Scotus, Quaestiones super Praedicamenta Aristotelis 11, hg. von Robert Andrews u.a., St. Bonaventure, NY, 1999, S. 343-354, bes. S. 350: ,[...] tunc videtur quod logicus, considerans per se intentiones et numerum generalissimorum tantum sub hac ratione - scilicet qua eis haec intentio,generalissimum ‘ possit applicari -, deberet dicere quod plura esse generalissima quam decem, quia plura istis sunt intentionalia quibus, in quantum intelliguntur, potest intentio attribui, licet metaphysicus, considerans ens inquantum ens, diceret ea tantum esse decem."

57 Ibid., S. 350: „Dicendum quod tantum sunt decem generalissima rerum [...].“ Dazu Pini, „Scotus on Deducing Aristotle's Categories“, op. cit., S. 28-33, und id., „Scotus's Realist Conception of the Categories. His Legacy to Late Medieval Debates“, in: Vivarium 43 (2005), S. 63-110, bes. S. 86-88. Nach Scotus sei es jedoch unmöglich, die Zehnzahl der Kategorien zu beweisen, da es sich hierbei um die ersten Arten des Seienden handle. 
Intention oder Imposition bezeichnet. ${ }^{58}$ Sie unterschieden sich nach Marsilius in der Art des Bezeichnens gänzlich von den Ersteren (,totaliter ratione differunt a terminis primae impositionis") und gehörten deshalb in eine eigene Kategorie der zweiten Intention oder Imposition, die den Kategorien der ersten Intention oder Imposition hinzugezählt und als ,praedicamentum signi“" oder „praedicamentum termini“" bezeichnet werden könne. ${ }^{59}$

Wo es um die Einführung der Kategorie des Zeichens ging, galt Marsilius nach dem Verständnis der spätmittelalterlichen Gelehrten als Schlüsselfigur. Sowohl Realisten als auch Nominalisten sahen diese Kategorie in Verbindung mit ihm. So schloss Johannes Versor seine Darstellung der Argumentation derer, die eine weitere Kategorie einführen wollten, mit den Worten: „et ob hoc posuit Marsilius praedicamentum signi. ${ }^{، 60}$ Auch in Ingolstadt galt diese Kategorie als ein besonderes Merkmal der Logik des Marsilius. Johannes Eck sprach in diesem Zusammenhang sogar von Marsilius und dessen Nachfolgern: „Marsilius cum sequacibus. ${ }^{\text {"61 }}$ Man kann also davon ausgehen, dass die Realisten in Ingolstadt, wenn sie sich kritisch über die Einführung einer elften Kategorie aussprachen, vor allem Marsilius und dessen Gefolgsleute im Blick hatten.

Einer dieser Gefolgsleute war der bereits erwähnte Johannes Parreut, der in seiner Schrift Exercitata veteris artis eine eigene Quaestio der Notwendigkeit einer elften Kategorie gewidmet hatte. ${ }^{62}$ Diese Quaestio wird im Nachfolgenden im Mittelpunkt stehen, denn sie deckt auf, aus welchen Gründen die Nominalisten in Ingolstadt die Einführung dieser Kategorie verteidigten und wie sie dies mit den Äußerungen des Aristoteles in Einklang brachten.

Parreut begann seine Quaestio mit einer Kritik an der realistischen Deutung der Kategorien, die er als „vana et falsa“ bezeichnete. ${ }^{63}$ Im Mittelpunkt stand das Kriterium, nach dem die Kategorien voneinander zu unterscheiden sind. Die Realisten gingen nach Johannes Parreut zu Unrecht von einer Ähnlichkeit zwischen den Arten der Prädikation einerseits und den Arten des Seins andererseits aus. Sie schlossen hieraus, dass jede Art der Prädikation eine besondere Art von Dingen (,res“) in der Wirklichkeit zum Ausdruck bringe, die der

58 Der Unterschied zwischen Begriffen der ersten und Begriffen der zweiten Imposition geht auf Boethius zurück, der ihn in seinem Kommentar zur Kategorienschrift ansprach; vgl. Boethius, In Categorias Aristotelis libri quatuor 1, op. cit., S. 159BC: ,[...] et est prima positio, ut nomina rebus imponerentur, secunda vero ut aliis nominibus ipsa nomina designarentur."

59 Marsilius von Inghen, Quaestiones veteris artis, Praedicamenta (Wien, Österreichische Nationalbibliothek, Cod. 5159), fol. $106^{\text {va }}$ : „Cum sint secundae intentionis seu impositionis, ideo oportet ipsa contineri sub genere generalissimo secundae intentionis seu impositionis. Et ita tandem erat repertum unum praedicamentum secundae intentionis seu impositionis et hoc potest nominari signi vel termini [...].“

60 Johannes Versor, Quaestiones super totam veterem artem, Praedicamenta, op. cit., fol. $29^{\mathrm{va}}$.

61 Johannes Eck, Aristotelis Dialectica, Categoriae 7, 3, Augsburg 1516, fol. $61^{\text {va }}$ : „[...] existimat Marsilius cum sequacibus, omnes secundas intentiones reponi in proprio praedicamento undecimo, quod praedicamentum signi appelletur." Zu Johannes Eck und dessen Bedeutung als Lehrer in Ingolstadt vgl. Arno Seifert, Logik zwischen Scholastik und Humanismus. Das Kommentarwerk Johann Ecks (Humanistische Bibliothek I/31), München 1978.

62 Johannes Parreut, Exercitata, op. cit., fol. $\mathrm{Si}^{\mathrm{v}}-\mathrm{Svi}^{\mathrm{r}}$ : „Utrum tantum decem sunt praedicamenta vel utrum praedicamentum signi ultra illa decem sit ponendum."

$63 \mathrm{Ibid}$., fol. Siir: „[...] relinquo illam opinionem antiquorum tanquam vanam et falsam.“ 
Sache und dem Wesen nach (,realiter et essentialiter") von anderen Dingen verschieden seien. ${ }^{64}$

Parreut kennzeichnete diese Lehre als die allgemeine Meinung aller Realisten („communis opinio omnium realistarum") ${ }^{65}$ Diese Charakterisierung galt für ihn jedoch nicht absolut, denn im weiteren Verlauf seiner Quaestio widerlegte er die realistische Lehre, indem er sich auf Thomas von Aquin stützte. Thomas habe in der Summa theologiae gelehrt, so Parreut, dass ein und derselbe Gegenstand auf verschiedene Weisen im Denken aufgefasst werden könne. Unterschiede im Sprechen und Denken über die Wirklichkeit bezeugen also nicht immer Unterschiede in der Wirklichkeit selbst. Aufgrund dieser Einsicht behaupteten die Nominalisten - Parreut sprach hier von „,communis via moderna“-, dass die zehn Kategorien des Aristoteles nur auf drei verschiedenen Arten von Gegenständen in der Wirklichkeit beruhen, und zwar der Substanz, der Qualität und der Quantität. Die Nominalisten und nicht die Realisten, so gab Parreut zu verstehen, folgten also der Lehre des Thomas von Aquin ${ }^{66}$

Aus diesem Rückgriff auf Thomas wird verständlich, weshalb die Nominalisten in Ingolstadt den Gegensatz zwischen beiden Wegen, wie die Realisten ihn versuchten herauszustellen, als unwichtig betrachteten und behaupten konnten, sie seien genauso gut in der Lage, die Texte von Thomas von Aquin und Johannes Versor in ihren Veranstaltungen zu behandeln wie die Realisten. ${ }^{67}$ Der Realismus, wie er sich vom Nominalismus zu unterscheiden suchte, sei nur eine Karikatur seiner selbst, die über ihre eigenen Quellen nicht im Klaren sei, so Parreut.

Wenn auch die Darstellung der Lehre der Realisten, wie Parreut sie hier gab, wohl aus argumentativen Gründen vereinfacht war, so entsprach sie dennoch den in seiner Zeit gängigen Vorstellungen. Ein ähnliches Bild entwirft die berühmte Verteidigungsrede der Pariser

$64 \mathrm{Ibid} .$, fol. $\mathrm{Si}^{\mathrm{v}}:$, [...] in hoc decipiuntur antiqui quod estimant tot debere esse res vel modos essendi rei quot sunt modi intelligendi vel modi significandi rerum.“ Und ibid.: ,[...] volunt quod realiter et essentialiter sint distincta [sc. praedicamenta, $\mathrm{MH}$ ] ita quod per ista decem praedicamenta importentur diversae res quarum una non est alia."

65 Ibid., fol. $\mathrm{Si}^{\mathrm{v}}$.

$66 \mathrm{Ibid}$., fol. $\mathrm{Si}^{\mathrm{v}}-\mathrm{Sii}^{\mathrm{r}}$ : ,[...] una res potest multis modis intelligi et significari. Ideo bene dicit sanctus Thomas in prima parte quaestione septuagesimasexta in solutione tertii argumenti: ,Non oportet secundum diversas rationes vel intentiones logicas, quae consequuntur modum intelligendi, diversitatem in rebus accipere, quia ratio idem et unum secundum diversos modos apprehendere potest.' Et ideo secundum communem viam modernam tantum tria praedicamenta ponunt in numerum, id est important significata realiter distincta ab invicem constituentia numerum, et hoc quo ad aliquas species eorum et non quo ad omnes, ut substantia, quantitas et qualitas." Es handelt sich hier um ein wörtliches Zitat aus Thomas von Aquin, Summa theologiae I, 76, 3, ad 4 (Opera omnia, Bd. V), Rom 1889, S. 221. Eine ähnliche Argumentation, jedoch ohne Verweis auf Thomas, schreibt Bartholomaeus Arnoldi von Usingen in einer Disputation aus dem Jahre 1497 den moderni zu; vgl. Bartholomaeus von Usingen, Quaestio de quiditate quantitatis continuae in disputatione de quolibet Erffordie A.D. 1497 determinata, hg. von Hans-Ulrich Wöhler, in: Bochumer philosophisches Jahrbuch für Antike und Mittelalter 6 (2001), S. 137-195, bes. S. 184: „Quare moderni negant illam: ,Quecumque distinguuntur predicamentaliter, distinguuntur realiter', loquendo de rebus substratis intentionibus predicamentalibus, quia eadem res potest substerni pluribus talibus propter aliam et aliam rationem considerandi.“Parreut sprach also zu Recht von einer „,communis via moderna“.

67 Siehe den Verweis in Anm. 50 oben. 
Nominalisten aus dem Jahre $1474 .^{68}$ Auch in dieser Schrift wurde pauschal behauptet, die Realisten gingen von einer Übereinstimmung zwischen Denken und Sein aus: „Reales autem res multiplicatas esse contendunt secundum multiplicationem terminorum. ${ }^{609}$ Der Grund, weshalb dies zur gängigen Vorstellung wurde, ist nicht leicht zu bestimmen. Es könnte sein, dass die Nominalisten gerne am Bild von der Übereinstimmung zwischen Sein und Denken festhielten, da Gerson in mehreren seiner Schriften die Annahme dieser Übereinstimmung als Ursache der Häresie von Hieronymus von Prag diagnostiziert hatte. Als Quelle dieser Lehre hob Gerson die These von Johannes Duns Scotus und Franciscus de Mayronis von der ,distinctio formalis a parte rei“ hervor. ${ }^{70}$ Es ließe sich die Argumentation von Parreut dann so verstehen, dass er die Realisten als Anhänger der genannten Lehre darstellte, um damit zum Ausdruck zu bringen, dass sie verurteilt werden müsse und unmöglich wahr sein könne.

Es ist aber auch möglich, dass im späten Mittelalter die Scotisten immer mehr an Einfluss gewannen und sie deshalb als die wichtigsten Vertreter der Realisten galten. ${ }^{71}$ Diese Möglichkeit trifft im Fall von Parreut wohl eher zu. Es ist nämlich auffallend, wie dieser seine Lehre der elften Kategorie mit Verweisen auf Scotus und Franciscus de Mayronis zu stützen versuchte. Franciscus de Mayronis habe gezeigt, so Parreut, dass die Zehnzahl der Kategorien von Aristoteles an keiner Stelle bewiesen wurde, und Scotus habe in seinem Kommentar zu den Sentenzen behauptet, dass die zweiten Intentiones und die ,entia rationis" in einer gesonderten Kategorie untergebracht werden sollten, und zwar in einer anderen als in den von Aristoteles genannten. ${ }^{72}$ Diese Linie sei von den moderni fortgeführt worden,

\footnotetext{
68 Der Anlass dieser Verteidigungsrede, das Edikt Ludwigs XI. gegen den Nominalismus, war in der Handschrift München, Universitätsbibliothek, $2^{\circ}$ Cod. ms. 482, enthalten. Vgl. Ehrle, Der Sentenzenkommentar des Peters von Candia, op. cit., S. 330.

69 Ibid., S. 322-326 (Edition der Verteidigungsrede), bes. S. 322. Die Beschreibung, die die Pariser Nominalisten von ihrer eigenen Vorgehensweise geben, ist ganz in Übereinstimmung mit dem, was Johannes Parreut und Bartholomaeus von Usingen oben in Anm. 66 als kennzeichnend für die Auffassung der moderni behauptet hatten; vgl. ibid., S. 322: „Illi Doctores Nominales dicti sunt qui non multiplicant res principaliter sign[ific] atas per terminos secundum multiplicationem terminorum.“

70 Vgl. Kaluza, Les querelles doctrinales à Paris, op. cit., S. 127-144, und Maarten J. F. M. Hoenen, „Modus loquendi Platonicorum. Johannes Gerson und seine Kritik an Plato und den Platonisten“, in: Stephen Gersh / Maarten J. F. M. Hoenen (Hgg.), The Platonic Tradition in the Middle Ages. A Doxographic Approach, Berlin 2002, S. 325-343.

71 In der frühen Neuzeit gehörte der Skotismus zu den bedeutendsten philosophischen Schulen; vgl. Felix Bak, „Scoti schola numeriosior est omnibus aliis simul sumptis“, in: Franciscan Studies 16 (1956), S. 144165, und Roger Ariew, Descartes and the Last Scholastics, Ithaca 1999, S. 39-57, bes. S. 45.

72 Johannes Parreut, Exercitata, op. cit., fol. Siii : „[...] Franciscus Mayronis dicit quod illa conclusio [sc. tantum decem sunt praedicamenta terminorum primae impositionis in genere, $\mathrm{MH}$ ] non possit evidenter ratione demonstrari sicut aliqui praeintendunt, sed oportet ita credere et praesupponere tamquam verum." Und ibid.: ,[...] in quarto Sententiarum distinctione prima [...] vult [Scotus, $\mathrm{MH}$ ] intentiones secundas esse in aliquo praedicamento et etiam entia rationis, sed non in aliquo decem praedicamentorum ab Aristotele positorum.“ Vgl. Johannes Duns Scotus, In librum IV Sententiarum 1, 2 (Opera omnia, Bd. VIII), Lyon 1639, Nachdruck Hildesheim 1968, S. 55-63, bes. S. 61-62. Für die Stelle bei Franciscus de Mayronis siehe oben Anm. 53.
} 
so Parreut, und sie hätten dazu eine besondere Kategorie bestimmt, die „praedicamentum signi“" genannt werde. ${ }^{73}$

Ein ähnlicher Verweis auf Scotus findet sich bei Johannes Eck, der eine Generation nach Parreut in Ingolstadt tätig war. Scotus habe darauf hingedeutet, dass die ,intentiones logicae" als eine eigene Art aufzufassen seien, obwohl er nicht den Schritt getan habe, sie mit einer eigenen Kategorie zu klassifizieren. ${ }^{74}$

Betrachtet man die Stelle aus dem Sentenzenkommentar des Scotus, auf die Parreut und Eck verwiesen, dann ist die Sache nicht so deutlich, wie beide vorgeben. Scotus sprach dort nicht von Kategorien, sondern nur von Definitionen im Hinblick auf Begriffe der zweiten Intention. Diese Begriffe haben eine eigene Definition, durch die sie von Begriffen der ersten Intention unterschieden sind: ,intentiones secundae sunt per se definibiles. ${ }^{675}$ Dass sie deshalb in eine eigene Kategorie gehören, wurde von Scotus nicht behauptet, auch nicht an anderen Stellen, sondern in seinem Kommentar zur Ars vetus nur referiert. Es waren jedoch diese Überlegungen, die spätere Scotisten zu der Überlegung veranlassten, ob zur Erfassung dieser ,intentiones secundae“ nicht eine eigene Kategorie erforderlich sei. So hob Petrus Tartaretus hervor, dass die zweiten Intentionen nicht in den üblichen zehn Kategorien untergebracht seien und deshalb womöglich zu einem eigenen ,praedicamentum entis rationis“ zählten. ${ }^{76}$

Mit solchen Verweisen auf Scotus und Franciscus de Mayronis setzte Parreut seine Strategie fort, die er bereits zuvor angewendet hatte. Er zeigte auch hier, dass die Lehre der Nominalisten keine Neuerung darstellte, sondern auf Überlegungen der Realisten zurückging, auch wenn diese nicht die Konsequenzen aus ihren eigenen Überlegungen gezogen hatten.

Obwohl Parreut auf die Realisten verwies, folgte er bei der Bestimmung der elften Kategorie den Vorgaben des Marsilius. Die Begriffe der zweiten Imposition unterschieden sich in der Art des Bezeichnens von den Begriffen der ersten Imposition, insofern sie als Zeichen eines Zeichens, und nicht als Zeichen der extramentalen Wirklichkeit galten. Damit war nach Parreut das wichtigste Kriterium für die Einführung einer neuen Kategorie erfüllt, dass es sich nämlich bei diesen Begriffen um eine eigene Weise des Bezeichnens handle. ${ }^{77}$

73 Johannes Parreut, Exercitata, op. cit., fol. Siii : „[...] illud praedicamentum baptizatur a Modernis et vocatur ,praedicamentum signi‘.“

74 Johannes Eck, Dialectica, Categoriae 7, 3, op. cit., fol. $62^{\text {ra }}$ : „Et adverte quod Scotus etiam innuit praedicamentum signi in quarto, distinctione prima, quaestione secunda, ubi testatur intentiones logicas non significare entia realia, et tamen non habeant sua genera, quamvis non ita expresse astruat intentiones secundas in alioquo poni praedicamento [...].“

75 Johannes Duns Scotus, In librum IV Sententiarum 1, 2, 8, op. cit., S. 61-62.

76 Petrus Tartaretus, Expositio super textu logices Aristotelis, Praedicamenta, Poitiers 1493/1494 (Freiburg UB, Ink $4^{\circ}$ D 1607 at) fol. cii ${ }^{\mathrm{v}}:,[\ldots]$ res secundae intentionis non sunt in aliquo decem praedicamentorum, licet possint poni in praedicamento entis rationis." Johannes Eck verwies auf die Stelle bei Petrus Tartaretus; siehe Johannes Eck, Dialectica, Categoriae 7, 3, op. cit., fol. 62a: „Ita et Tartaretus ait secundas intentiones reponi in praedicamento entis rationis." Petrus Tartaretus war seit 1484 als Magister an der Artesfakultät von Paris tätig. Seine Kommentare zu Aristoteles und Petrus Hispanus wurden oft gedruckt. Zu ihm siehe Charles H. Lohr, „Medieval Latin Aristotle Commentaries“, in: Traditio 28 (1972), S. 281-396, bes. S. 372376.

77 Johannes Parreut, Exercitata, op. cit., fol. Siii": „[...] signum habet modum significandi distinctum ab omnibus aliis praedicamentis, ut notum est. Ergo est ponendum.“ 
Aber es gab noch ein weiteres Kriterium, das in der Debatte über die Notwendigkeit der Einführung einer elften Kategorie eine Rolle gespielt hat, und zwar die ontologische Natur dessen, was in einer Kategorie überhaupt erfasst werden soll. Den Realisten aus Ingolstadt zufolge bezogen sich die Kategorien auf Dinge außerhalb des menschlichen Geistes, in dem Sinne wie Thomas im obigen Zitat von ,ens extra animam“ gesprochen hatte. Sie stützen sich dabei unter anderem auf die Metaphysik des Aristoteles, in der ausgeführt wurde, dass die Kategorien das reale Sein klassifizierten. ${ }^{78}$ Eine solche Deutung wurde auch von anderen Realisten vertreten. So kritisierte zum Beispiel Johannes Versor die Lehre des Marsilius, indem er hervorhob, dass die Begriffe und Wörter kein Sein im eigentlichen (,ens verum et perfectum"), sondern lediglich im abgewandelten Sinne hatten (,ens secundum quid“), denn sie seien vom menschlichen Geist hervorgebracht, der kein wirkliches, sondern nur gedachtes Seiendes produzieren könne. Deshalb könne nach Johannes Versor von einer eigenen Kategorie des Zeichens keine Rede sein. ${ }^{79}$

Parreut war in seiner Quaestio ausführlich auf diese Kritik eingegangen. Seine Antwort ist aufschlussreich dafür, wie die spätmittelalterlichen Nominalisten über die ontologische Natur der Begriffe dachten. Parreut zufolge handelt es sich bei Begriffen entgegen der Meinung der Realisten um Seiendes im eigentlichen Sinne. Begriffe sind nämlich nicht zusammengesetzt und alles nicht Zusammengesetzte sei als Seiendes ,per se“ zu betrachten. Was Aristoteles dem Sein im eigentlichen Sinne entgegenstellt habe, seien keine Begriffe gewesen, sondern Sätze, die aus Begriffen zusammengesetzt sind und deshalb kein selbstständiges Sein hätten. Die Realisten, so Parreut, deuteten Aristoteles demnach in verkehrter Weise. Sie gingen davon aus, dass nach Aristoteles nur Seiendes außerhalb des menschlichen Geistes als Seiendes im wirklichen Sinne bezeichnet werden könne. Aber das sagte Aristoteles nicht. Er sprach nicht von „ens extra animam“, wie Thomas behauptete, sondern lediglich von „ens per se“ und „ens proprie dictum“, und damit war alles Seiende gemeint, das nicht zusammengesetzt war, also auch Begriffe, wie Parreut mit einem Verweis auf Buridan unterstrich. ${ }^{80}$

So blieb noch der Einwand, dass Aristoteles eine solche Kategorie nicht erwähnt hatte.

78 Vgl. Ehrle, Der Sentenzenkommentar des Peters von Candia, op. cit., S. 335: „Et patet in libro predicamentorum eiusdem et in septimo Metaphisice, ubi dividit ens extra animam in decem predicamenta." Siehe auch Johannes Parreut, Exercitata, op. cit., fol. Siii": „[...] secundum realistas [...] ista praedicamenta decem sunt tantum entium extra animam."

79 Johannes Versor, Quaestiones super totam veterem artem Aristotelis, op. cit., fol. $30^{\mathrm{ra}}$ : „[...] non significant [sc. intentiones secundae, $\mathrm{MH}$ ] ens verum et perfectum, sed solum ens secundum quid, quod est ens rationis tantum, quasi a ratione causatum." Ähnlich argumentierten die Thomisten in Köln; vgl. Copulata in veterem artem secundum viam doctoris Thomae de Aquino, Praedicamenta, op. cit., fol. $48^{\mathrm{r}}$ : ,[...] auctoritas illorum [sc. modernorum, qui ponunt praedicamentum signi, $\mathrm{MH}$ ] non est recipienda ut vera, quia praedicamenta debent esse entia realia iuxta intentionem Philosophi, qui dividit ens reale in decem praedicamenta." 80 Johannes Parreut, Exercitata, op. cit., fol. Sv ${ }^{\mathrm{v}}$ : ,[...] quamvis antiqui communiter ita allegant Aristotelem, tamen ita non reperitur. Sed Aristoteles in quinto Metaphysicae in capitulo ,ens' bene dividit ens per se in praedicamenta; sed signa etiam sunt entia per se. Et in sexto Metaphysicae Aristoteles dividit ens proprie acceptum in praedicamenta; sed ens proprie dictum non vocatur ens extra animam tantum, sicut antiqui volunt, sed ens proprie dictum est ens incomplexum, ut Buridanus ibidem vult [...].“ Ich habe im 6. Buch des Metaphysikkommentars von Buridan, wie es vorliegt in der Ausgabe Kommentar zur Aristotelischen Metaphysik, Paris 1518, Nachdruck Frankfurt am Main 1964, keine Stelle finden können, die unmittelbar die Behauptung Parreuts stützt. 
Aber auch das stellte für Parreut keinen Grund dar, von deren Einführung abzusehen. Aristoteles hatte sich in seinen Erörterungen nur mit den Kategorien beschäftigt, die sich auf die Begriffe der ersten Imposition bezogen, so argumentierte er. Die Tatsache, dass Aristoteles zehn Kategorien aufgelistet hatte, schloss also nicht aus, dass es noch eine zusätzliche Kategorie gab, die sich auf Begriffe der zweiten Imposition bezog, auch im Sinne des Aristoteles. Die ,auctoritas negativa“ hatte für Parreut also keine Geltung. ${ }^{81}$ Es handelt sich bei der elften Kategorie lediglich um eine verbale Neuerung, insofern Aristoteles diese Kategorie nicht erwähnt hatte. Aber sie war keine Neuerung in dem Sinne, dass sie im Widerspruch zu den Ausgangspunkten des Aristoteles stand.

Kommen wir zum Schluss und fassen wir die Ergebnisse in einigen Punkten zusammen.

1. Die Realisten in Ingolstadt kritisierten die Nominalisten, nicht nur deshalb, weil diese Begriffe einführten, die Aristoteles nicht verwendet hatte, wie das „praedicamentum signi“, sondern auch, weil die Nominalisten die Texte des Aristoteles in einer Art und Weise deuteten, die mit dessen Lehre im Widerspruch stand, sogar dort, wo sie am Wortlaut festhielten. Was hiermit gemeint war, hat die vorangehende Analyse deutlich gezeigt. Die Nominalisten hatten eine andere Auffassung von der Beziehung zwischen Denken und Sein und von der ontologischen Bestimmung der Begriffe im menschlichen Geist. ${ }^{82}$ Diese Unterschiede wirkten sich in der Auslegung der aristotelischen Kategorienlehre unmittelbar aus, sowohl bei der Deutung der von Aristoteles genannten zehn Kategorien als auch bei der Frage, ob unter den von Aristoteles gegebenen Bedingungen eine zusätzliche Kategorie angenommen werden kann. Debatten über die Beziehung zwischen Denken und Sein und die Natur der Begriffe sind nicht typisch für das 15 . Jahrhundert, sondern wurden bereits in der Zeit von Thomas, Scotus und Ockham geführt. Es ist aus diesem Grund, dass sich beide Parteien auf Autoritäten des 13. und 14. Jahrhundert beriefen und darauf beharren konnten, auch wenn sie diese nicht immer in gleicher Weise interpretierten. ${ }^{83}$

2. In ihrer Deutung des Aristoteles hielten die Realisten sich an die Vorgaben von Albertus Magnus, Thomas von Aquin und Duns Scotus und stellten diese den Auslegungen von

81 Johannes Parreut, Exercitata, op. cit., fol. $\mathrm{Sv}^{\mathrm{r}}$ : , [...] locus ab auctoritate negativa non valet, quia Aristoteles et alii auctores loquuntur tantum de praedicamentis terminorum primae impositionis."

82 So vertrat Johannes Eck, der sich in seinen Schriften immer wieder auf nominalistische Autoren wie Johannes Buridan und Marsilius von Inghen stützte, die Auffassung, dass die Begriffe im menschlichen Geist reales Sein haben; siehe Johannes Eck, Dialectica, Praedicabilia 1, 3, op. cit., fol. 14 ${ }^{\mathrm{va}}$ : „Conceptus sive universalis sive singularis habet esse verum reale et subiectivum in anima sicut reale accidens." $\mathrm{Er}$ bezeichnete diese Lehre, ibid., fol. $14^{\mathrm{rb}}$, als die „opinio iam communis apud Neotericos“ und zitierte, ibid., fol. 14 ${ }^{\mathrm{va}}$, als Quellen die Quodlibeta von Ockham und einen Brief des Gerson, den er als „De laudibus Bonaventurae“ anführte - denselben Brief, auf den Parreut verwiesen hatte (s.o. Anm. 26). Vor allem dieser Brief ist historisch von Bedeutung, da er die Lehre, dass die Begriffe kein Sein im eigentlichen Sinne haben (also die Lehre, wie die Realisten sie nach Parreut vertraten) ausdrücklich zurückweist; siehe Johannes Gerson, Gerson à un Frère Mineur (Lyon, 7 décembre 1426), op. cit., S. 279-280: „Tradunt nonnulli, unde mirandum videtur, quod conceptus et entia rationis non sunt vere res nec entia [...]; cum tamen accidentia animae $[. .$.$] sint entia longe perfectiora quam albedo vel nigredo [...].“$

$83 \mathrm{Zu}$ den früheren Debatten vgl. Maarten J. F. M. Hoenen, „Being and Thinking in the Correctorium fratris Thomae and the Correctorium corruptorii Quare. Schools of Thought and Philosophical Methodology", in: Jan A. Aertsen / Kent Emery, Jr. / Andreas Speer (Hgg.), Nach der Verurteilung von 1277. Philosophie und Theologie an der Universität von Paris im letzten Viertel des 13. Jahrhunderts (Miscellanea Mediaevalia 28), Berlin 2001, S. 417-435. 
Buridanus und Marsilius von Inghen entgegen, wie sie von den Nominalisten verteidigt wurden. Aber ganz so einfach war die Sache nicht. Aus den Darstellungen Parreuts geht hervor, dass die Nominalisten den Gegensatz anders beurteilten. Diesem zufolge sind die Lehren des Thomas und Scotus sehr wohl mit denen von Buridanus und Marsilius in Einklang zu bringen, auch dort wo es um die Beziehung zwischen Sein und Denken und die ontologische Natur der Begriffe geht. Auch anderswo wurden solche Querverbindungen gelegt. So rechnete der Heidelberger Nominalist Stephan Hoest Duns Scotus zu den Urhebern der via moderna. Zudem hat die Forschung festgestellt, dass es signifikante Übereinstimmungen gibt zwischen den Nominalisten und den Thomisten in Köln. ${ }^{84}$ Die Gegensätze, wie die Realisten in Ingolstadt sie hervorhoben, wurden von den Nominalisten dann auch als nicht zutreffend betrachtet. Sie interpretierten diese als taktische Konstrukte der Realisten, mit denen diese sich von den Nominalisten zu unterscheiden suchten. Aus dieser Perspektive ist auch klar, weshalb die Nominalisten den Autoritätsglauben der Realisten so stark anprangerten. Die Realisten hielten an ihren Autoren fest, ohne zu versuchen, deren Ansätze weiterzuentwickeln. Aber auch hier könnten die Realisten sich mit der Feststellung verteidigen, dass die Nominalisten sich zwar auf die Texte des Thomas und Scotus stützten, diese aber in verkehrter Weise auslegten. Diese Konstellation erschwert das Verständnis des Wegestreits, der gerade ein Streit über die Quellen war, die bei der Auslegung des Aristoteles heranzuziehen waren, und sie zeigt, dass die Art und Weise, wie mit diesen Quellen umgegangen werden sollte, von den verschiedenen Parteien ganz anders wahrgenommen wurde und deshalb auch immer nur unter Berücksichtigung verschiedener Ausgangspunkte gedeutet werden kann.

3. Bemerkenswert ist die Rolle, die Marsilius von Inghen in der Debatte über die elfte Kategorie gespielt hat. Er galt als deren Urheber, auch wenn darauf hingewiesen wurde, dass andere bereits in diese Richtung gedacht hatten. Auch andere Ergänzungen der aristotelischen Begrifflichkeit schrieb man ihm zu, wie die Annahme einer fünften Art der Qualität, wo Aristoteles nur vier unterschieden hatte.$^{85}$ Es handelt sich in beiden Fällen um Auffassungen, von denen man im 15. Jahrhundert behauptete, sie gehörten zur ,communis opinio modernorum“. Dabei wurde von einigen Autoren auch herausgestellt, dass Ockham eine andere Lehre vertreten hatte. ${ }^{86}$ In Ingolstadt verteidigte Johannes Parreut diese neuen Lehren. Wie am Anfang gesagt, hob er zu Beginn seines Werkes zwar hervor, dass Ockham mit Peter von Ailly und Gerson zu den wichtigsten Autoren zähle. Wo es aber um die beiden genannten Punkte ging, zog er nicht Ockham, sondern die Neuerungen des Marsilius vor. Der Nominalismus in Ingolstadt war also kein Ockhamismus, auch wenn die Realisten es

84 Vgl. Stephan Hoest, Reden und Briefe. Quellen zur Geschichte der Scholastik und des Humanismus im 15. Jahrhundert (Humanistische Bibliothek. Abhandlungen und Texte II/3), hg. von Frank Baron, München 1971, S. 176, und Henk A. G. Braakhuis, „Heymeric van de Velde (a Campo), denker op een kruispunt van wegen. De ,logische' kwestie uit zijn Problemata inter Albertum et Sanctum Thomam", in: Algemeen Nederlands Tijdschrift voor Wijsbegeerte 75 (1983), S. 13-24, bes. S. 18-20.

85 Johannes Parreut, Exercitata op. cit., fol. Qiii: , „[...] propter istud vel plura alia motiva Marsilius ponit speciem quintam qualitatis, quae non habet nomen incomplexum impositum propter penuriam vocabulorum, sed potest circumloqui [...].“ Vgl. Ehrle, Der Sentenzenkommentar des Peters von Candia, op. cit., S. 336: „[...] item circa species qualitatis, ubi Nominales plures species nominant, quas Aristoteles non posuit.“

86 So Johannes Eck bei der Frage, ob eine elfte Kategorie anzunehmen sei; vgl. Johannes Eck, Dialectica, Categoriae 7, 3, op. cit., fol. $61^{\mathrm{va}}$. 
versuchten so darzustellen, sondern schöpfte aus unterschiedlichen Quellen, wie dies auch an anderen Universitäten der Fall war. In Freiburg zum Beispiel wurde die via moderna gelegentlich zwar als „via Ockham“ bezeichnet. ${ }^{87}$ In Wirklichkeit aber ging der dortige Unterricht hauptsächlich auf Texte von Buridan und Marsilius von Inghen zurück. ${ }^{88}$

4. Die obigen Betrachtungen erlauben es, den Ausgang des Streits zu verstehen. In den Jahren 1519/1520 bestimmten die Statuten der Artesfakultät, dass die Begriffe „moderni“, „antiqui“, „realistae“ und „,nominalistae“ nicht länger verwendet werden dürften. Alle Magister und Studenten sollten nur noch mit einem allen gemeinsamen Namen bezeichnet werden: „artistae“. Damit war der Wegestreit in Ingolstadt, zumindest dem Namen nach, beendet. ${ }^{89}$ Diese Entwicklung hatte ihren Anfang schon früher genommen. Seit 1515 wurde an der Fakultät eine gründliche Reform des Unterrichts mit dem Ziel durchgeführt, den Auseinandersetzungen der beiden Wege ein Ende zu bereiten. ${ }^{90}$ Betrachtet man die Lehrbücher, die angefertigt wurden, um dieser Reform Rechnung zu tragen, dann profilieren diese sich zwar im Sinne der Statuten von 1519/1520 nicht länger als einer bestimmten Richtung zugehörig; aus dem Inhalt geht jedoch hervor, dass die nominalistische Linie, wie Parreut sie vertreten hatte, fortgesetzt wurde, wenn auch mit Modifizierungen. ${ }^{91}$ Diese Linie hatte bereits zuvor gezeigt, dass sie beide Traditionen miteinander zu verbinden verstand. Insofern lag es in der Natur der Dinge, dass der Nominalismus als Sieger aus dem Streit hervorgehen würde. Zugleich wird deutlich, dass unabhängig vom Verschwinden der Namen, die ver-

87 Universitätsarchiv Freiburg (UAF), Protokollbuch der Philosophischen Fakultät 1460-1531, B 38/9, fol. $140^{\mathrm{r}}$ (1495) und fol. $129^{\mathrm{r}}(1496)$.

88 Mindestens seit 1470 wurden bei der Lektüre der Werke des Aristoteles fast ausschließlich die Kommentare von Buridanus und Marsilius benutzt; siehe UAF, Protokollbuch der Philosophischen Fakultät 14601531, B 38/9, fol. 43 ${ }^{\mathrm{r}}$. Dazu Friedrich Schaub, „Die älteste Stipendienstiftung an der Universität Freiburg i. Br. und ihr Stifter Konrad Arnolt von Schorndorf", in: Zeitschrift der Gesellschaft für Beförderung der Geschichts-, Altertums- und Volkskunde von Freiburg, dem Breisgau und den angrenzenden Landschaften 38 (1925), S. 53-88, bes. S. 67-68. Von Bedeutung für das mittelalterliche Verständnis der Quellen ist die (irrige) Bemerkung Johannes Ecks in dessen in Freiburg entstandener Schrift Bursa Pavonis, dass Marsilius ein Zeitgenosse und „socius“ von Buridanus gewesen sei; vgl. Johannes Eck, Bursa pavonis. Logices exercitamenta, Straßburg 1507, fol. Eiii": „Secunda opinio et magistri Marsilii, qui fuit contemporarius et socius ipsius Buridani." Diese falsche Auffassung hat sich bis in die moderne Historiographie erhalten.

89 Vgl. Gabriel, „Via antiqua and via moderna and the Migration of Paris Students“, op. cit., S. 476-477, und Prantl, Geschichte der Ludwig-Maximilians-Universität, op. cit., Bd. II, S. 154-162, bes. S. 155-156 (Statuten).

90 Vgl. Seifert, Logik zwischen Scholastik und Humanismus, op. cit., S. 7-9.

91 Die Dialectica des Johannes Eck wurde im Rahmen dieser Reform angefertigt; vgl. Seifert, Logik zwischen Scholastik und Humanismus, op. cit., S. 7-13 und S. 58-73. Johannes Eck bezeichnet Ockham in dieser Schrift nicht länger als „Guillelmus noster“ oder „doctor noster“, wie er es fast durchweg in seiner zehn Jahre zuvor gedruckten Schrift Bursa Pavonis getan hatte (vgl. Johannes Eck, Bursa pavonis. Logices exercitamenta, op. cit., fol. $\mathrm{Cvi}^{\mathrm{r}}$ und Eiiii ${ }^{\mathrm{r}}$. Dennoch wurde Ockham sehr oft zusammen mit Buridanus, Marsilius, Holcot, Gregorius von Rimini und Johannes Parreut genannt. Auch wird auf Albertus, Bonaventura und Scotus Bezug genommen, jedoch sind die Verweise auf diese Autoren deutlich seltener. Als Thomisten treten die Magister der Kölner Bursa Montana hervor, die jedoch in der Regel kritisiert werden; siehe etwa Johannes Eck, Dialectica, Praedicabilia 1, 3, fol. $14^{\text {ra }}$ : „Male ergo negat mons Gymnicus Coloniense universale esse genus, quia non dicatur univoce de praedicabilibus.“ 
schiedenen Deutungen des Aristoteles noch immer eine Rolle spielten, um bald im Zuge der Reformation in neuer Gestalt wieder in Erscheinung zu treten. ${ }^{92}$

92 Vgl. etwa Charles H. Lohr, „Metaphysics and Natural Philosophy as Sciences. The Catholic and Protestant Views in the Sixteenth and the Seventeenth Centuries“, in: Constance Blackwell / Sachiko Kusukawa (Hgg.), Philosophy in the Sixteenth and Seventeenth Centuries. Conversations with Aristotle, Aldershot 1999, S. 280-295. 